\title{
Molecular Mechanisms of Altered Bile Acid Homeostasis in Organic Solute Transporter-Alpha Knockout Mice
}

\author{
Tian Lan Jamie Haywood Anuradha Rao Paul A. Dawson \\ Department of Internal Medicine, Section on Gastroenterology and Department of Pathology, \\ Wake Forest University School of Medicine, Winston-Salem, N.C., USA
}

\section{Key Words}

Enterohepatic circulation $\cdot$ Intestine $\cdot$ Liver $\cdot$ Cholesterol $\cdot$ Villus

\begin{abstract}
Background/Aims: Mutations in the apical sodium-dependent bile acid transporter (SLC1OA2) block intestinal bile acid absorption, resulting in a compensatory increase in hepatic bile acid synthesis. Inactivation of the basolateral membrane bile acid transporter (OST $\alpha$-OST $\beta$ ) also impairs intestinal bile acid absorption, but hepatic bile acid synthesis was paradoxically repressed. We hypothesized that the altered bile acid homeostasis resulted from ileal trapping of bile acids that act via the farnesoid $X$ receptor (FXR) to induce overexpression of FGF15. To test this hypothesis, we investigated whether blocking FXR signaling would reverse the bile acid synthesis phenotype in Ost $\alpha$ null mice. Methods: The corresponding null mice were crossbred to generate Ost $\alpha F x$ r double-null mice. All experiments compared wild-type, Ost $\alpha, F x r$ and Ost $\alpha$ Fxr null littermates. Analysis of the in vivo phenotype included measurements of bile acid fecal excretion, pool size and composition. Hepatic and intestinal gene and protein expression were also examined. Results: Ost $\alpha \mathrm{Fxr}$ null mice exhibited increased bile acid fecal excretion and pool size,
\end{abstract}

and decreased bile acid pool hydrophobicity, as compared with Ost $\alpha$ null mice. Inactivation of FXR reversed the increase in ileal total FGF15 expression, which was associated with a significant increase in hepatic Cyp7a1 expression. Conclusions: Inactivation of FXR largely unmasked the bile acid malabsorption phenotype and corrected the bile acid homeostasis defect in Ost $\alpha$ null mice, suggesting that inappropriate activation of the FXR-FGF15-FGFR4 pathway partially underlies this phenotype. Intestinal morphological changes and reduced apical sodium-dependent bile acid transporter expression were maintained in $\mathrm{Ost}^{-/-} \mathrm{Fxr}^{-/}$mice, indicating that FXR is not required for these adaptive responses.

Copyright $\odot 2011$ S. Karger AG, Basel

\section{Introduction}

Bile acids are synthesized from cholesterol in the liver and secreted with bile into the small intestine to facilitate absorption of dietary lipids. Bile acids are almost quantitatively reclaimed by the intestine ( $>95 \%$ ) and transported back to the liver to be resecreted into bile. Efficient intestinal reabsorption and hepatic extraction of bile acids enables a very effective recycling and conservation mechanism that largely restricts these cytotoxic deter-

\section{KARGER}

() 2011 S. Karger AG, Basel

Fax +4161306 1234

E-Mail karger@karger.ch

www.karger.com
Accessible online at: www.karger.com/ddi
Paul A. Dawson

Department of Internal Medicine, Section on Gastroenterology

Wake Forest University School of Medicine, Medical Center Blvd.

Winston-Salem, NC 27157-1048 (USA)

Tel. +1 336716 4633, E-Mail pdawson@wfubmc.edu 
gents to the intestinal and hepatobiliary compartments $[1,2]$. Since the early 1990s, many of the hepatic and intestinal transporters that function to maintain the enterohepatic circulation of bile acids have been identified $[3,4]$.

In the ileum, the apical sodium-dependent bile acid transporter (Asbt or ibat; gene symbol Slc10a2) mediates the uptake of bile acids from the gut lumen across the apical brush border membrane of the enterocyte. Considering the large flux of bile acids across the ileal enterocyte (approx. $25 \mathrm{mg} /$ day in mice; approx. 20-30 g/day in humans) and that there is a dedicated apical carrier, it is likely that a dedicated basolateral bile acid transporter is also involved. More recently, the unusual heteromeric transporter Ost $\alpha$-Ost $\beta$ was identified and shown to transport a variety of solutes, including bile acids and steroids [5]. Subsequently, several lines of evidence emerged that support a role for Ost $\alpha$-Ost $\beta$ in the basolateral membrane transport of bile acids in the intestine, liver and kidney [6-14].

Targeted inactivation of Ost $\alpha$ in mice was associated with significantly decreased ileal absorption of bile acids, as demonstrated using everted gut sacs or by intraluminal administration of bile acids $[12,15]$. However, whereas hepatic bile acid synthesis and fecal bile acid excretion is typically elevated in patients with Asbt mutations [16] or in $A s b t^{-/-}$mice $[17,18]$, Ost $\alpha^{-/-}$mice exhibited decreased hepatic Cyp7a1 expression and no increase in fecal bile acid excretion. In this study, we tested the hypothesis that the farnesoid X receptor (FXR) is involved in the dysregulated bile acid metabolism and adaptive responses observed in $\mathrm{Ost}^{-/-}$mice.

\section{Results}

\section{Phenotype of $\mathrm{Ost}^{-/-} \mathrm{Fxr}^{-/-}$Mice}

$\mathrm{Ost}^{-/-}$mice (in a mixed C57BL/6J-129/SvEv background) were generated as previously described by Rao et al. [12]. $\mathrm{Fxr}^{-/-}$mice were purchased from the Jackson Laboratory (stock No.: 004144, strain: B6;129X(FVB)$\left.\mathrm{Nr} 1 \mathrm{~h} 4^{\mathrm{tm} 1 \mathrm{Gonz}} / \mathrm{J}\right)$. The corresponding null mice were crossbred to generate $\mathrm{Ost}^{-/-} \mathrm{Fxr}^{-/-}$mice. The experiments were performed using wild-type, Ost $\alpha^{-/-}, \mathrm{Fxr}^{-/-}$and $\mathrm{Ost}^{-/-} \mathrm{Fxr}^{-/-}$littermates in a mixed background, which were maintained on a rodent-chow diet. The $\mathrm{Osta}^{-/-} \mathrm{Fxr}^{-/-}$ mice were viable and fertile. There was no obvious prenatal mortality in these backgrounds as crosses between heterozygous mice produced the predicted Mendelian distribution of wild-type and mutant genotypes. There were no significant differences in body weight or liver weight between the adult wild-type and Ost $\alpha^{-/-}$mice or between the $\mathrm{Fxr}^{-1-}$ and Ost $\alpha^{-/} \mathrm{Fxr}^{-/-}$mice. As previously reported $[12,15]$, the most significant morphological finding was a longer and heavier small intestine in Ost $\alpha^{-/}$mice as compared with wild-type littermates. Specifically, the small intestine of adult male $\mathrm{Ost}^{-/-}$mice (3 months of age) was approximately $18 \%$ longer and $46 \%$ heavier; similar results were observed for female Ost $\alpha^{-/-}$ mice versus wild-type mice. Inactivation of FXR in $\mathrm{Ost}^{-/-}$mice largely restored the small intestine to the wild-type length, but did not reverse the increase in the intestinal weight. The increased intestinal weight was particularly evident in distal small intestine of Ost $\alpha^{-/-}$ and $\mathrm{Ost}^{-/-} \mathrm{Fxr}^{-/-}$mice.

Quantitative morphometric analyses were also performed to examine the effects of Ost $\alpha$ inactivation on small intestine. Histological evaluation revealed little change in proximal small intestine, but it did show a significantly thickened mucosa in ileum. Villus width and crypt depth were all significantly increased in ileum of Ost $\alpha^{-/-}$mice as compared with wild-type or $\mathrm{Fxr}^{-/-}$littermates. FXR deficiency did not reverse this phenotype in $O s t \alpha^{-/-}$mice, suggesting that bile acid signaling via this nuclear receptor is not responsible for the intestinal hyperplasia. These morphological changes were not accompanied by an increase in inflammatory cell infiltration. In addition, analysis of mRNA expression revealed no increases in expression of proinflammatory genes such as TNF $\alpha$ or IL-1 $\beta$, or genes involved in ER stress such as Xbox-binding protein-1 (Xbp1) or activating transcription factor-6 (Atf6) in ileum of Ost $\alpha^{-/-}$or $\mathrm{Ost}^{-1-} \mathrm{Fxr}^{-/-}$mice versus wild-type or $\mathrm{Fxr}^{-/-}$mice. The gene expression profile was consistent with the histological absence of intestinal inflammation and the lack of diarrhea in Ost $\alpha^{-/}$ and $\mathrm{Osta}^{-/-} \mathrm{Fxr}^{-/-}$mice.

\section{Fecal Bile Acid Excretion and Pool Size in}

$$
\text { Ost } \alpha^{-/-} \mathrm{Fxr}^{-/-} \text {Mice }
$$

Inactivation of Ost $\alpha$ was predicted to induce bile acid malabsorption by blocking enterocyte basolateral membrane bile acid transport, a phenotype similar to that observed for a block in intestinal apical uptake of bile acids [16-18]. However, as previously reported [12, 15], fecal bile acid excretion was not increased in $\mathrm{Ost}^{-/-}$mice, despite an apparent block in intestinal bile acid absorption and a greater than $50 \%$ decrease in the bile acid pool size. Although this decrease in bile acid pool size was similar to that observed in $\mathrm{Asbt}^{-/-}$mice, the Ost $\alpha^{-/-}$mice exhibited a disproportionate decrease in the taurocholate frac- 
tion, yielding a more hydrophilic bile acid pool that is enriched in tauro- $\beta$-muricholate. This phenotype is in contrast with the $A s b t^{-/-}$mice, where elevated hepatic bile acid synthesis via the Cyp7a1/Cyp8b1 pathway results in a bile acid pool that is significantly enriched in taurocholate.

The phenotype in Ost $\alpha^{-/-}$mice is consistent with a paradoxical downregulation of hepatic bile acid synthesis via the Cyp7a1 pathway. In light of the central role of the FXR-FGF15-FGFR4 pathway in regulation of Cyp7a1 and hepatic bile acid synthesis [19, 20], we hypothesized that the altered bile acid homeostasis in $\mathrm{Ost}^{-/-}$was secondary to increased activation of FXR and induction of FGF15 expression in ileum. In support of that hypothesis, FXR deficiency in $\mathrm{Ost}^{-/-}$mice unmasked the underlying bile acid malabsorption phenotype, increasing fecal bile acid excretion by almost threefold in $\mathrm{Osta}^{-/-} \mathrm{Fxr}^{-/-}$versus Ost $\alpha^{-/-}$mice.

The bile acid pool size was also partially restored following inactivation of FXR, increasing almost twofold in male and female Ost $\alpha^{-/-} \mathrm{Fxr}^{-/-}$versus Ost $\alpha^{-/-}$mice. However, the bile acid pool size was still reduced in $\mathrm{Ost}^{-/-} \mathrm{Fxr}^{-/-}$versus $\mathrm{Fxr}^{-/-}$mice and wild-type mice, demonstrating that the increased hepatic bile acid synthesis cannot fully compensate for the decreased intestinal bile acid absorption as a result of inactivation of Ost $\alpha$. FXR deficiency increased the proportion of taurocholate in the bile acid pool, as reflected by an increased calculated hydrophobicity of the bile acid pool in $\mathrm{Osta}^{-/-} \mathrm{Fxr}^{-/-}$versus $O s t \alpha^{-/-}$mice. Overall, inactivation of FXR in Ost $\alpha^{-/-}$mice resulted in a bile acid phenotype that was more similar to the $A s b t^{-/-}$than wild-type mice.

\section{Expression of Hepatic and Intestinal Genes Involved} in Bile Acid Metabolism in $\mathrm{Ost}^{-/-} \mathrm{Fxr}^{-/-}$Mice

In agreement with the bile acid fecal excretion and pool size results, inactivation of FXR was associated with a dramatic induction of Cyp7al mRNA expression in the Ost $\alpha^{-/-}$mice. Hepatic expression of Cyp7a1 mRNA was increased almost eightfold in Ost $\alpha^{-/-} \mathrm{Fxr}^{-/-}$mice as compared to $O s t \alpha^{-/-}$mice. A similar trend was observed for hepatic Cyp8b1 mRNA expression, which was increased almost fourfold. The gene expression changes appeared to be restricted to the classical bile acid biosynthetic pathway, as there were no changes in hepatic mRNA expression for Cyp27al, a major enzyme in the alternative pathway.

Analysis of the gradient of FGF15 mRNA expression along the cephalocaudal axis of the small intestine revealed no major differences between the four genotypes, and FGF15 mRNA expression was largely restricted to distal small intestine. Contrary to the predicted mechanism for the decreased hepatic Cyp7al expression, ileal FGF15 mRNA expression (when normalized to expression of the housekeeping gene cyclophilin) was not significantly increased in Ost $\alpha^{-/-}$versus wild-type mice. However, total ileal FGF15 mRNA expression was increased almost threefold in Ost $\alpha^{-/-}$versus wild-type mice when the intestinal hyperplasia was considered and gene expression was normalized for the increased RNA content of the tissue. Inactivation of FXR dramatically reduced ileal cellular and total FGF15 mRNA expression in agreement with previous studies demonstrating an essential role of FXR in regulating FGF15 expression [1921]. In $\mathrm{Ost}^{-/-} \mathrm{Fxr}^{-1-}$ mice, ileal total FGF15 mRNA expression was reduced by approximately $90 \%$ as compared to $\mathrm{Ost}^{-/-}$mice.

Although these results indicate that FXR is essential for the increased intestinal FGF15 expression, the finding that the FGF15 mRNA expression per cell was not increased suggests that bile acids do not accumulate within the enterocyte or that the cell is hyporesponsive to the bile acid load. In order to investigate those potential mechanisms, the cellular expression of other FXR-responsive genes was investigated. Ileal cellular mRNA expression of the FXR target genes Ibabp and Ost $\beta$ was significantly decreased in ileum from $\mathrm{Ost}^{-/-}$mice. This apparent decrease in FXR activity may be secondary to the decreased bile acid pool size in Ost $\alpha^{-/-}$mice. However, another potential mechanism is decreased Asbt expression, which would function to limit the uptake and accumulation of cytotoxic bile acids in ileal enterocytes. In fact, ileal Asbt expression was found to be reduced by approximately $50 \%$ at the mRNA level and greater than $90 \%$ at the protein level in $\mathrm{Ost}^{-/-}$mice. Notably, the dramatic reduction in Asbt mRNA and protein expression was maintained in $\mathrm{Ost}^{-/-} \mathrm{Fxr}^{-/-}$mice, indicating that FXR is not required for this adaptive response.

\section{Discussion}

In this study, we examined the role of FXR in the altered regulation of bile acid metabolism in Ost $\alpha^{-/-}$mice. In summary, we demonstrated that FXR is essential for repression of Cyp7a1 expression in Ost $\alpha^{-/-}$mice. Genetic inactivation of FXR in Ost $\alpha^{-/-}$mice increased bile acid synthesis, reversed shifts in bile acid pool composition and restored intestinal cholesterol absorption. However, FXR deficiency failed to reverse the intestinal morpho- 


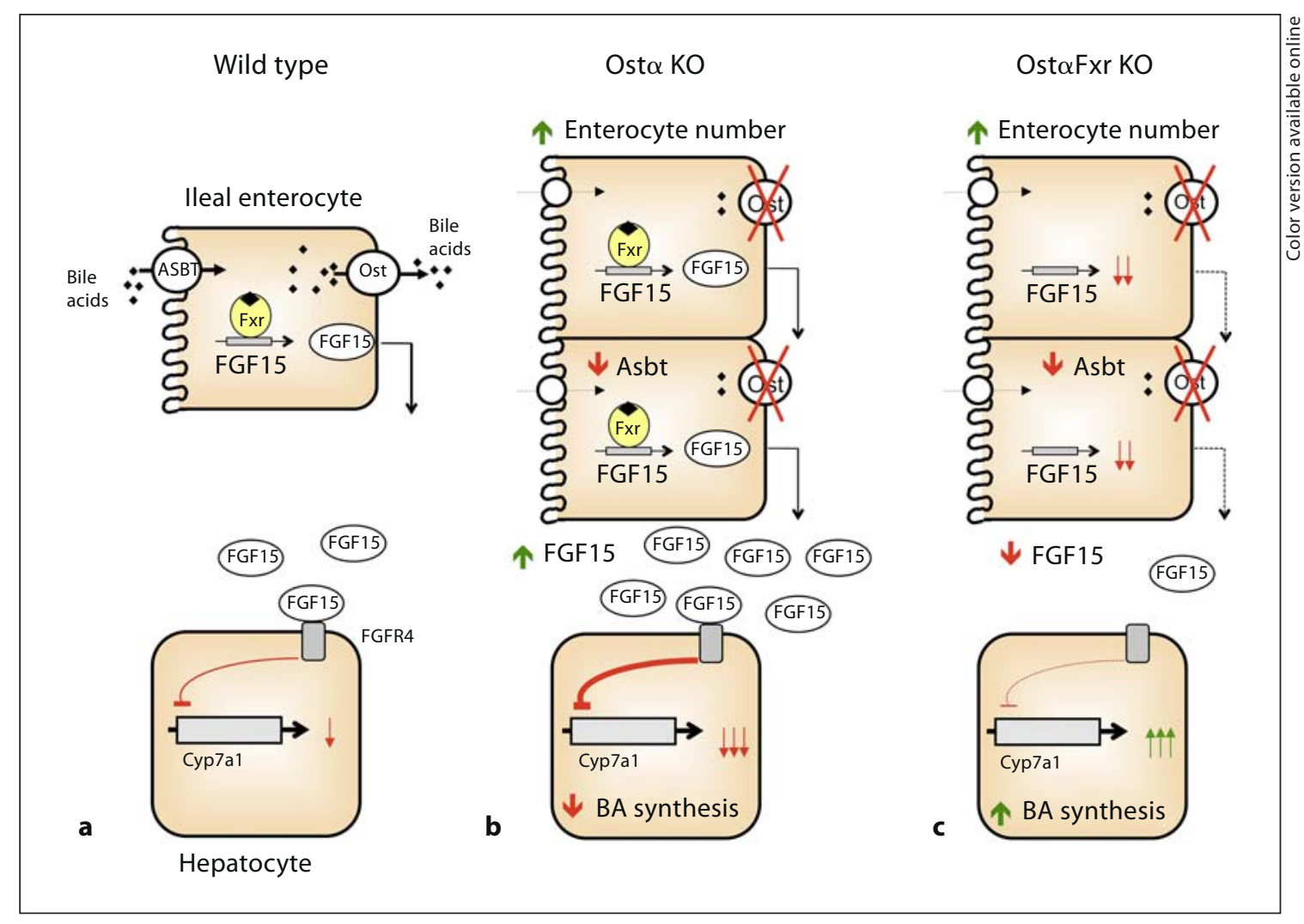

Fig. 1. Model depicting the regulation of ileal FGF15 synthesis and hepatic bile acid (BA) synthesis in wild-type, Ost ${ }^{-/-}$and Ost ${ }^{-1-} \mathrm{Fxr}^{-1-}$ mice. Schematic model depicting the differential regulation of Cyp7al. a Bile acids are absorbed from the intestinal lumen via Asbt and activate FXR to induce FGF15 expression. Ileal-derived FGF15 acts on the liver via its receptor, FGFR4, to repress Cyp7a1 expression and bile acid synthesis. b A reduction in the bile acid pool size and decreased Asbt expression reduces the accumulation of bile acids in the ileal enterocytes. However, ileal hyperplasia leads to an increase in the number of FGF15expressing enterocytes. As a result, the total intestinal FGF15 expression is increased and signals to repress hepatic Cyp7a1 expression and bile acid synthesis. $\mathrm{c}$ Inactivation of FXR does not reverse the downregulation of Asbt expression or intestinal morphological changes in the Ost $\alpha^{-/-}$mice. However, inactivation of FXR significantly reduces ileal FGF15 expression. As a result, the total intestinal FGF15 expression is reduced, and hepatic Cyp7al expression and bile acid synthesis is increased. logical changes in $\mathrm{Ost}^{-/-}$mice. Moreover, it is not an enhanced activation of FXR that drives the increase in intestinal FGF15 expression, but rather a complex adaptive response that increases the ileal enterocyte population. In fact, a decrease in the bile acid pool size and significantly reduced ileal Asbt expression may function to protect the enterocytes in $\mathrm{Ost}^{-/-}$mice from accumulating potentially cytotoxic levels of bile acids. These findings are consistent with a critical but limited role of FXR in the adaptive responses engaged to compensate for the loss of Ost $\alpha$ in mice. The current model depicting the hypothesized mechanism for regulation of bile acid homeostasis in wild-type, Ost $\alpha^{-/-}$and $\mathrm{Ost}^{-/-} \mathrm{Fxr}^{-/-}$mice is shown in figure 1.
These results generally support a significant role for Ost $\alpha$-Ost $\beta$ in intestinal basolateral bile acid transport. However, many questions remain to be answered, particularly with regard to the complex intestinal adaptive response in $\mathrm{Ost \alpha}^{-/-}$mice. This intestinal phenotype is not a general response to bile acid malabsorption, as similar changes in intestinal morphology were not observed in $A s b t^{-/-}$or $\mathrm{Mrp}^{-/-}$mice $[17,18,22]$. Nor does the intestinal adaptation appear to be a direct response to bile acid toxicity, as neither bile acid accumulation nor intestinal inflammation were observed in Ost $\alpha^{-/-}$mice. The finding that inactivation of FXR failed to reverse the intestinal adaptive response indicates that the changes are not mediated through increased bile acid signaling via this nu-

Dig Dis 2011;29:18-22 
clear receptor. As such, other mechanisms such as the accumulation of non-bile acid substrates may be involved. Ost $\alpha$-Ost $\beta$ has been shown to transport other solutes, such as steroids and steroid sulfates [23], and enhanced signaling due to accumulation of these substrates may be involved in promoting intestinal growth in $\mathrm{Ost}^{-/-}$mice.

In conclusion, activation of the FXR-FGF15-FGFR4 pathway is essential for the altered bile acid homeostasis associated with genetic ablation of the intestinal basolateral bile acid transporter Ost $\alpha$-Ost $\beta$. Surprisingly, a complex adaptive response that increases the ileal enterocyte population largely accounts for the increase in total intestinal FGF15 expression in Ost $\alpha^{-/-}$mice rather than increased bile acid accumulation and FXR activation.

\section{Acknowledgments}

This project was supported by NIH DK047987 and an American Heart Association Mid-Atlantic Affiliate grant-in-aid (to P.A.D.). A.R. is supported by a National Research Service Award (F32 DK079576) from the NIDDK.

\section{Disclosure Statement}

P.A.D. has served on the Scientific Advisory Board and owns equity in XenoPort Inc. He has also served as a consultant to GlaxoSmithKline, Isis Pharmaceuticals, Sanofi-Aventis and Bristol-Myers Squibb. T.L., J.H. and A.R. have no conflicts of interest to report.

\section{References}

$\checkmark 1$ Hofmann AF, Hagey LR: Bile acids: chemistry, pathochemistry, biology, pathobiology, and therapeutics. Cell Mol Life Sci 2008;65: 2461-2483.

2 Hofmann AF: Bile acids: trying to understand their chemistry and biology with the hope of helping patients. Hepatology 2009; 49:1403-1418.

-3 Alrefai WA, Gill RK: Bile acid transporters: structure, function, regulation and pathophysiological implications. Pharm Res 2007; 24:1803-1823.

4 Dawson PA, Lan T, Rao A: Bile acid transporters. J Lipid Res 2009;50:2340-2357.

$\rightarrow 5$ Seward DJ, Koh AS, Boyer JL, Ballatori N: Functional complementation between a novel mammalian polygenic transport complex and an evolutionarily ancient organic solute transporter, OSTalpha-OSTbeta. J Biol Chem 2003;278:27473-27482.

-6 Lee H, Zhang Y, Lee FY, Nelson SF, Gonzalez FJ, Edwards PA: FXR regulates organic solute transporters alpha and beta in the adrenal gland, kidney, and intestine. J Lipid Res 2006;47:201-214.

7 Frankenberg T, Rao A, Chen F, Haywood J, Shneider BL, Dawson PA: Regulation of the mouse organic solute transporter alpha-beta, Ostalpha-Ostbeta, by bile acids. Am J Physiol Gastrointest Liver Physiol 2006; 290:G912-G922.

8 Landrier JF, Eloranta JJ, Vavricka SR, Kullak-Ublick GA: The nuclear receptor for bile acids, FXR, transactivates human organic solute transporter-alpha and -beta genes. Am J Physiol Gastrointest Liver Physiol 2006;290:G476-G485.
9 Boyer JL, Trauner M, Mennone A, et al: Upregulation of a basolateral FXR-dependent bile acid efflux transporter OSTalpha-OSTbeta in cholestasis in humans and rodents. Am J Physiol Gastrointest Liver Physiol 2006;290:G1124-G1130.

10 Dawson PA, Hubbert M, Haywood J, et al: The heteromeric organic solute transporter alpha-beta, Ostalpha-Ostbeta, is an ileal basolateral bile acid transporter. J Biol Chem 2005;280:6960-6968.

11 Ballatori N, Christian WV, Lee JY, et al: OSTalpha-OSTbeta: a major basolateral bile acid and steroid transporter in human intestinal, renal, and biliary epithelia. Hepatology 2005;42:1270-1279.

12 Rao A, Haywood J, Craddock AL, Belinsky MG, Kruh GD, Dawson PA: The organic solute transporter alpha-beta, Ostalpha-Ostbeta, is essential for intestinal bile acid transport and homeostasis. Proc Natl Acad Sci USA 2008; 105:3891-3896.

13 Soroka CJ, Mennone A, Hagey LR, Ballatori N, Boyer JL: Mouse organic solute transporter alpha deficiency enhances renal excretion of bile acids and attenuates cholestasis. Hepatology 2010;51:181-190.

14 Dawson PA, Hubbert ML, Rao A: Getting the mOST from OST: Role of organic solute transporter, OSTalpha-OSTbeta, in bile acid and steroid metabolism. Biochim Biophys Acta 2010;1801:994-1004.

15 Ballatori N, Fang F, Christian WV, Li N, Hammond CL: Ostalpha-Ostbeta is required for bile acid and conjugated steroid disposition in the intestine, kidney, and liver. Am J Physiol Gastrointest Liver Physiol 2008; 295:G179-G186.
16 Oelkers P, Kirby LC, Heubi JE, Dawson PA: Primary bile acid malabsorption caused by mutations in the ileal sodium-dependent bile acid transporter gene (SLC10A2). J Clin Invest 1997;99:1880-1887.

17 Jung D, Inagaki T, Gerard RD, et al: FXR agonists and FGF15 reduce fecal bile acid excretion in a mouse model of bile acid malabsorption. J Lipid Res 2007;48:2693-2700.

18 Dawson PA, Haywood J, Craddock AL, et al: Targeted deletion of the ileal bile acid transporter eliminates enterohepatic cycling of bile acids in mice. J Biol Chem 2003;278: 33920-33927.

19 Inagaki T, Choi M, Moschetta A, et al: Fibroblast growth factor 15 functions as an enterohepatic signal to regulate bile acid homeostasis. Cell Metab 2005;2:217-225.

20 Kim I, Ahn SH, Inagaki T, et al: Differential regulation of bile acid homeostasis by the farnesoid X receptor in liver and intestine. J Lipid Res 2007;48:2664-2672.

21 Stroeve JH, Brufau G, Stellaard F, Gonzalez FJ, Staels B, Kuipers F: Intestinal FXR-mediated FGF15 production contributes to diurnal control of hepatic bile acid synthesis in mice. Lab Invest 2010;90:1457-1467.

22 Belinsky MG, Dawson PA, Shchaveleva I, et al: Analysis of the in vivo functions of Mrp3. Mol Pharmacol 2005;68:160-168.

23 Ballatori N, Li N, Fang F, Boyer JL, Christian WV, Hammond CL: OST alpha-OST beta: a key membrane transporter of bile acids and conjugated steroids. Front Biosci 2009; 14: 2829-2844. 